\title{
An Automated Device for Recording Peripheral Arterial Waveform
}

\author{
Tuukka Panula $^{1}$, Juuso Blomster ${ }^{2}$, Mikko Pänkäälä $^{1}$, Tero Koivisto $^{1}$, Matti Kaisti $^{1}$ \\ ${ }^{1}$ University of Turku, Department of Future Technologies, Turku, Finland \\ 2 Turku University Hospital, Turku, Finland
}

\begin{abstract}
The aim of the study was to develop an automated device for recording peripheral arterial pulse wave, in order to assess cardiovascular health. Recent studies have shown that photoplethysmography $(P P G)$ is a viable technique to measure peripheral pressure waveform. We developed a small motorized device that can measure pulse waveform from a finger. The device targets the distal transverse palmar arch (DTPA) artery using infrared wavelength PPG. Measurements were taken from healthy subjects $(n=8)$. The device was validated by performing HR detection and waveform analysis. The device was able to record high quality blood pressure calibrated arterial waveforms and detect beat-to-beat heart rate allowing the assessment of cardiovascular health status.
\end{abstract}

\section{Introduction}

The arterial pulse waveform holds a plethora of information giving a more accurate picture of a person's cardiovascular health compared to basic systolic and diastolic blood pressure values [17]. Traditionally physicians make a diagnosis based on the patient's blood pressure values and and usually do not consider the arterial waveform. Since the emergence of arterial tonometry and techniques enabling continuous recording of arterial waveform, more emphasis has been put to the pulse waveform analysis. Devices providing such analysis include Sphyghmocor (Atcor Medical, Australia), EndoPAT (Itamar Medical, Israel), various devices utilizing the Vascular Unloading Technique (VUT) e.g. Finapres NOVA (Finapres Medical Systems, Netherlands) and CNAP Monitor 500 (CNSystems, Germany) as well as modified oscillometric devices such as Arteriograph (Tensiomed, Hungary) and Mobil-O-Graph (IEM, Germany). [2], [16], [9], [19], [7], [6]

Photoplethysmographic waveform and its origins have recently been studied extensively [15]. It has been shown that PPG waveform correlates moderately with intra-arterial pressure readings [10]. Another recent study has shown PPG's viability in oscillometric blood pressure measurement along with a calibrated force sensor [3]. This indicated that amplitude of the PPG waveform behaves similarly to a traditional tonometric waveform when transmural pressure is varied.

\section{Methods}

\subsection{Hardware Description}

The system was built around the Arduino microcontroller platform equipped with an 8-bit Microchip AVR MCU (Microcontroller Unit) [12]. The system block diagram is depicted in Figure 1. A custom add-on shield was designed for interfacing the peripherals with the MCU. A unipolar stepper motor (28-BYJ48) and its driving Darlington transistor array were connected to a dedicated motor controller MCU (Microchip AVR ATtiny84) [13]. The motor controller was connected to the main MCU via a UART (Universal Asynchronous Receiver Transmitter) connection. A PPG sensor unit (Maxim MAX30101) and a force sensor were connected to the MCU via shared $\mathrm{I}^{2} \mathrm{C}$ (Inter-Integrated Circuit) bus [11]. The PPG module is capable of transmitting and receiving up to 3 different wavelengths $(880 \mathrm{~nm}, 660 \mathrm{~nm}, 537 \mathrm{~nm})$ but only the infrared $(880 \mathrm{~nm})$ wavelength was chosen for this study. Level shifting circuitry ensured that the voltage levels on the MCU and sensors were matched. The force sensor was constructed by modifying a barometric pressure sensor (Bosch Sensortech BMP180) by removing the metal enclosure and submerging the sensor chip under a urethane gel bulb [1]. The mechanics were 3D printed to house the electronics, sensors and the stepper motor. A linear motion press was used to apply controlled pressure on the finger. The PPG sensor PCB (Printed Circuit Board) was placed on top of the force sensor in order to simultaneously record optical pulse wave and the compression level.

\subsection{System Operation}

The control software was programmed and a simple GUI was developed (Graphical User Interface) on MATLAB. The device connects to a PC via USB and 


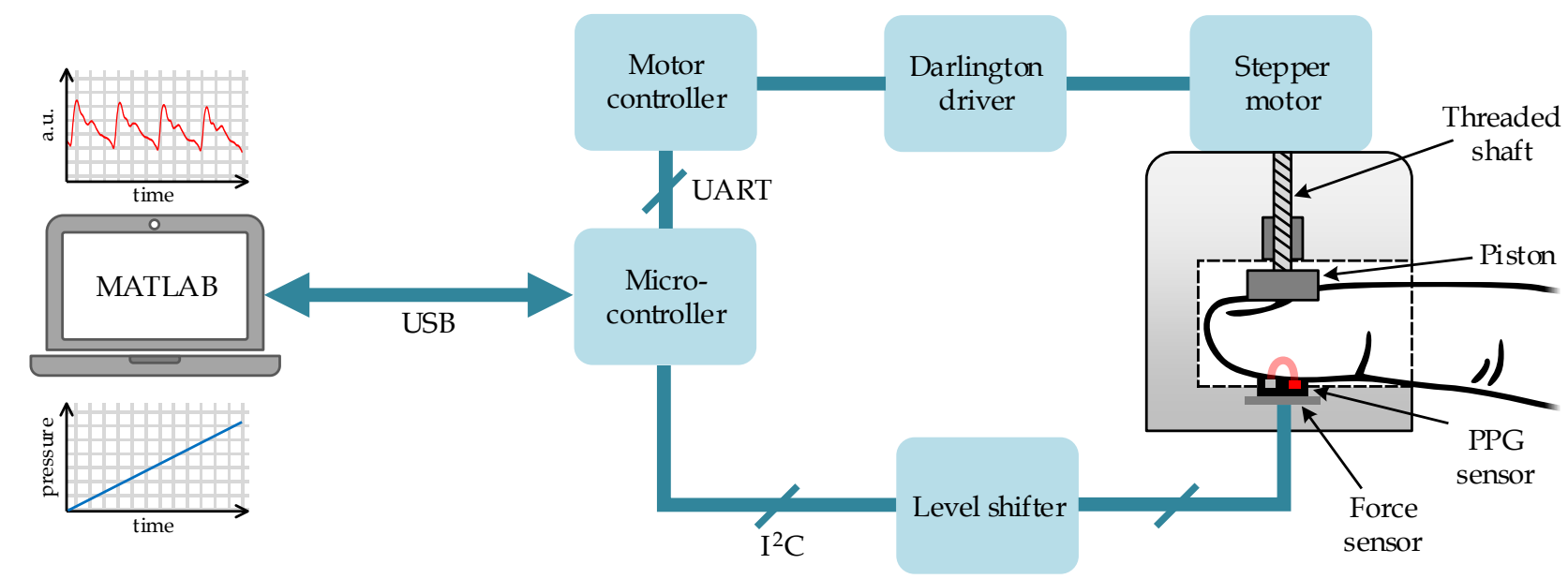

Figure 1. System block diagram.

provides a serial connection to send commands and receive sensor data from the device. When start is pressed the motor starts to compress the finger until the targeted artery (DTPA, distal tranverse palmar arch) is occluded and no pulsatile waveform can be seen. The pipeline for system operation is shown in Figure 2b). A bell-shaped oscillogram envelope is constructed by bandpass filtering $(1-10 \mathrm{~Hz})$, Hilbert transform and polynomial fitting respectively. Using the maximum of the envelope as a reference point, the device releases pressure until the corresponding compression level is achieved. In this point the transmural pressure equals zero and the external applied pressure equals MAP (Mean Arterial Pressure), leading to maximal pulse amplitude. The resulting waveform was calibrated with systolic and diastolic pressure readings acquired via standard brachial cuff oscillometry (Omron M3 Intellisense). Mean Arterial Pressure (MAP) was also calculated using: $M A P=$ $\frac{1}{3}(S B P-D B P)+D B P$.

The heart rate (HR) detection algorithm is depicted in Figure 2a) [8]. First an FIR (Finite Impulse Response) equiripple bandpass filter $(1-10 \mathrm{~Hz})$ was applied. The filtered signal was then convoluted with a triangular wavelet in order to smoothen the pulses. A multiscale-based peak detection algorithm as proposed by Scholkmann et al. was performed and median beat interval was calculated in order to get the HR [18].

\section{Results}

To verify the device's operation, 8 healthy subjects (1 female) were measured using the device and an automated blood pressure cuff $(S B P=126 \pm 13 \mathrm{mmHg}, D B P=$
$73 \pm 8 \mathrm{~mm} \mathrm{Hg}$ ). For demonstration purposes, HR was calculated for each subject. The HR detection was performed using a custom algorithm for evaluating the performance of the device. HR detection was successful $(T R P=100 \%, P P V=100 \%)$ in all subjects. The high detection rate is partly attributed to high quality signals.

Pulse morphology could be studied from the recorded arterial waveform. Figure 3 shows the measurement cycle along with the motor periods. Different transmural pressures and their impact on the resulting waveform were

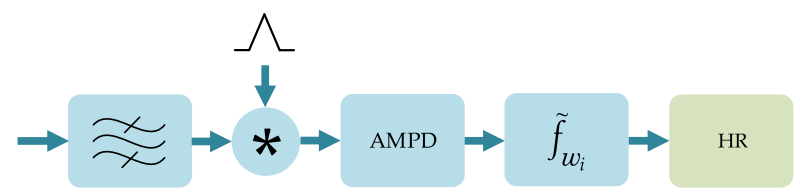

a)

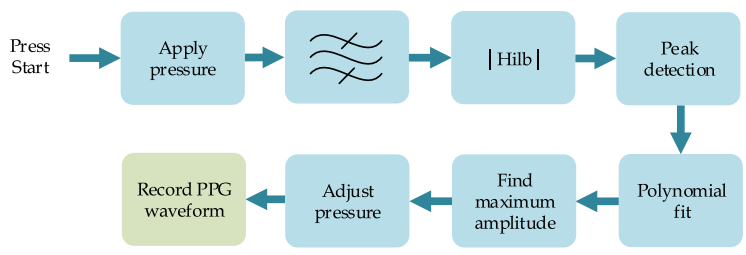

b)

Figure $2 . \quad$ a) Heart rate detection algorithm (bandpass filtering, convolution with triangular wavelet, multiscale-based peak detection, median beat interval. b) Pipeline of the automated measurement protocol. 


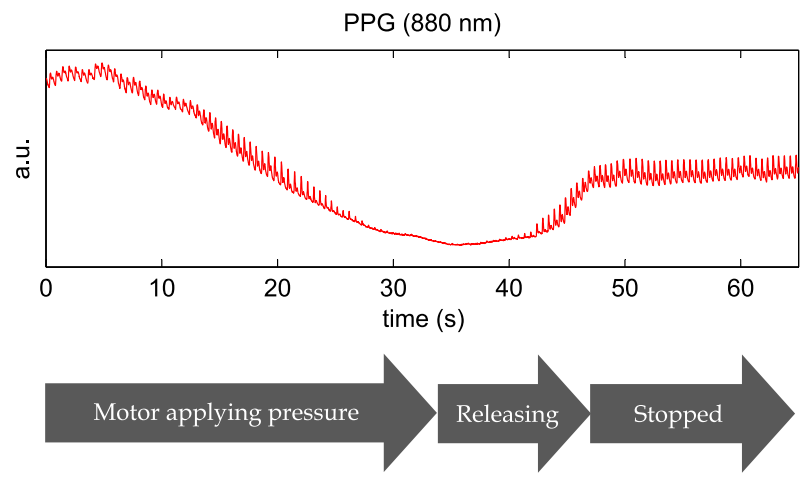

Figure 3. Approximately 1 minute of PPG recording. The bottom arrows indicate operation of the stepper motor controlling the compression stage. Waveform analysis is performed after the motor has stopped.

experimented with as seen in Figure 4c). This was done by recording pulse wave with negative $\left(P_{\text {ext }}<M A P\right)$, positive $\left(P_{\text {ext }}>M A P\right)$ and zero $\left(P_{\text {ext }} \approx M A P\right)$ transmural pressures. Negative transmural pressure resulted in a flattened waveform and positive in a sharp waveform with an exaggerated systolic peak. Maximum amplitude and best signal quality was achieved at zero transmural pressure. Diastolic peak $\left(P_{4}\right)$ and dicrotic notch $\left(P_{3}\right)$ were clearly visible. In addition to systolic peak and thus pulse pressure $(S B P-D B P)$, the late systolic peak (inflection point) could be extracted on most signals. Figure $4 \mathrm{~b}$ ) represents the formation of the late systolic peak by wave reflection caused by peripheral vessel resistance. The forward wave (I) is reflected back to the aorta due to high resistance arteries and veins producing the reflected wave (II). Note that the formation of $P_{3}$ and $P_{4}$ are not depicted here. The relation of the pulse pressure at the inflection point $\left(P P_{2}\right)$ and the systolic pulse pressure $\left(P P_{1}\right)$ is called peripheral augmentation index:

$$
p A I x=\frac{P P_{2}}{P P_{1}} \times 100(\%)
$$

This formula was used to calculate peripheral augmentation index for the subjects that showed a clear inflection point $(p A I x=85 \pm 8.5 \%)$. The larger the $p A I x$, the more resistance the pulse wave confronts when it travels to high resistance vessels. The amplification of the reflected wave happens due to various physiological (both neural and structural) processes, expecially when plaque starts forming in the arteries (atherosclerosis) and the vascular system loses its ability to adapt to static and dynamic pressure changes. Young people usually have a lower augmentation index than the elderly, indicating better vascular health. [14]
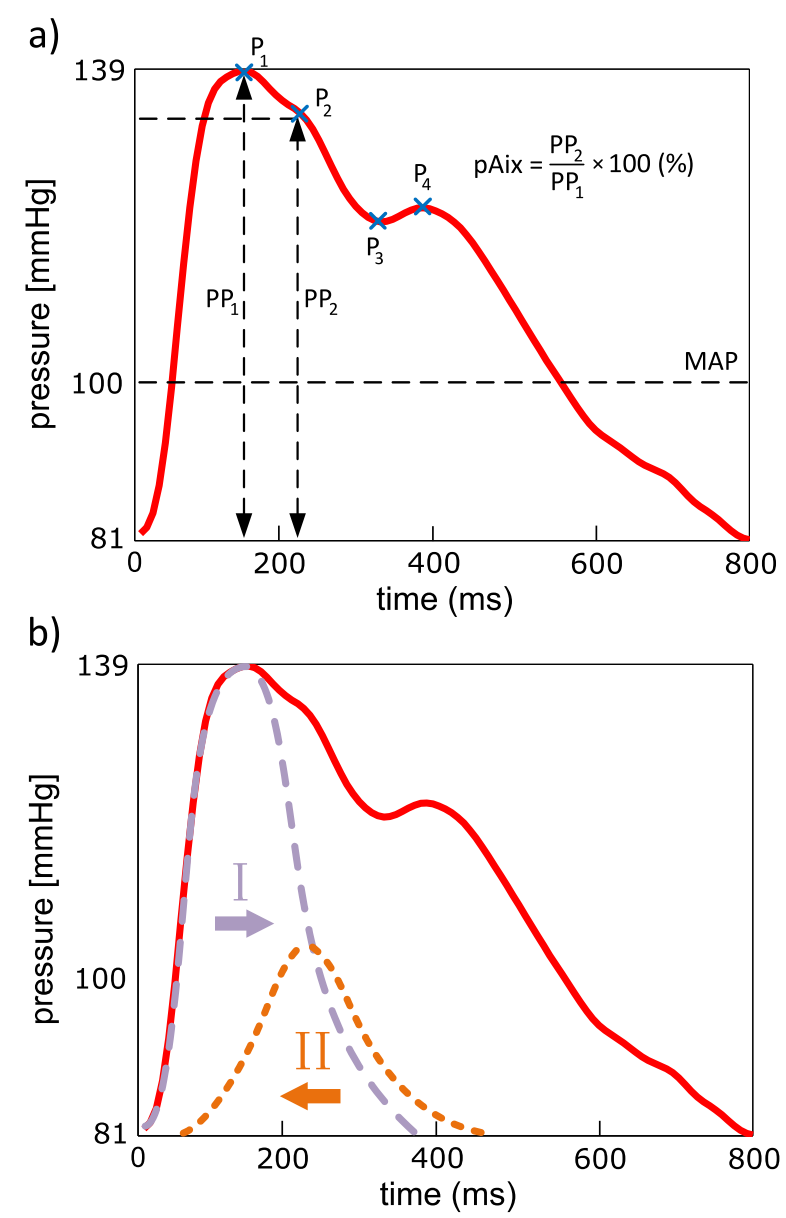

c)

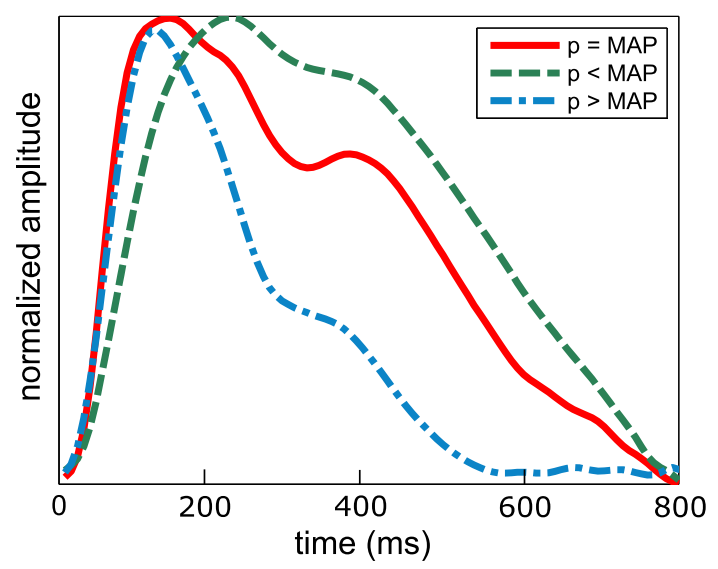

Figure 4. Pulse wave analysis. Top: arterial pulse calibrated to brachial blood pressures. $P_{1}$ and $P_{4}$ indicate the systolic and diastolic peaks respectively. $P_{2}$ shows the late systolic peak and $P_{3}$ shows the inflection point. Center: the same arterial waveform showing forward (I) and reflected (II) waves. Bottom: Pulse morphology analysis using different compression levels. Note that the amplitudes are normalized. 


\section{Discussion}

The device was able to record high quality blood pressure calibrated arterial waveforms and detect beat-to -beat heart rate allowing the assessment of the full peripheral waveform profile. Compared to devices using the Vascular Unloading Technique, the need for precise and fast switching pneumatics is eliminated which significantly reduces system complexity. The averaged pulse waveform could potentially be further used to derive stroke volume (SV) and cardiac output (CO) or transformed into aortic pressure waveform using a Generalized Transfer Function (GTF) [5], [4].

A further clinical cross validation study with a validated non-invasive blood pressure device or an intra-arterial reference would tell how the resulting waveform actually correlates with true arterial waveform. Additionally central blood pressure waveform could be recorded using a gold standard device such as Sphygmocor and a customized population based transfer function could be formulated.

\section{Acknowledgements}

The work of Tuukka Panula was funded by Business Finland (project ID: 2600455711).

\section{References}

[1] Bosch Sensortech, "BMP180 Digital pressure sensor," Datasheet, rev. 2.5, Apr 2013.

[2] M. Butlin et al., "Large artery stiffness assessment using sphygmoCor technology," Pulse, vol. 4, no. 4, pp. 180-192, Jun 2017.

[3] A. Chandrasekhar et al., "Smartphone-based blood pressure monitoring via the oscillometric finger-pressing method," Sci Transl Med, vol. 10, no. 431, Sep 2018.

[4] C. Chen et al., "Estimation of central aortic pressure waveform by mathematical transformation of radial tonometry pressure," Circulation, vol. 95, pp. 18271836, Apr 1997.

[5] J. Grensemann et al., "Cardiac output monitoring by pulse contour analysis, the technical basics of less-invasive techniques," Front Med, vol. 5, no. 64, Mar 2018.

[6] B. Hametner et al., "Oscillometric estimation of aortic pulse wave velocity: comparison with intra-aortic catheter measurements," Blood Press Monit, vol. 18, no. 3, pp. 173-176, Jun 2013.

[7] M. Illyes et al., "A new and fast screening method for measuring complex hemodynamical parameters and arterial stiffness non-invasively with a simple arm cuff," Am J Hyper, vol. 18, no. S4, p. 15A, May 2005.

[8] M. Kaisti et al., "Clinical assessment of a non-invasive wearable MEMS pressure sensor array for monitoring of arterial pulse waveform, heart rate and detection of atrial fibrillation," npj Digital Medicine, vol. 2, no. 39, May 2019.
[9] J. L. Kermode et al., "Comparison of the Finapres blood pressure monitor with intra-arterial manometry during induction of anaesthesia," Anaesth Intensive Care, vol. 17, no. 4, pp. 470-475, Nov 1989.

[10] G. Martinéz et al., "Can photoplethysmography replace arterial blood pressure in the assessment of blood pressure?," J Clin Med, vol. 7, no. 316, Sep 2018.

[11] Maxim Integrated, "MAX30101 high-sensitivity pulse oximeter and heart-rate sensor for wearable health," Datasheet, rev. 2, Oct 2018.

[12] Microchip Technology (Atmel), "ATMega329P 8-bit AVR microcontroller with $32 \mathrm{~K}$ bytes in-system programmable flash," Datasheet, rev. 7810D, Jan 2015.

[13] Microchip Technology (Atmel), "ATtiny24/44/84 8-bit AVR microcontroller with $2 / 4 / 8 \mathrm{~K}$ bytes in-system programmable flash," Datasheet, rev. 7701G, Feb 2015.

[14] H. Miyashita, "Clinical assessment of central blood pressure," Curr Hypertens Rev, vol. 8, no. 2, pp. 80-90, 2012.

[15] A. V. Moço et al., "New insights into the origin of remote PPG signals in visible light and infrared," Sci Rep, vol. 8, no. 8501, May 2018.

[16] M. Moerland et al., "Evaluation of the EndoPAT as a tool to assess endothelial function," Int J Vasc Med, vol. 2012, no. 904141, 8 pages, Feb 2012.

[17] M. F. O'Rourke et al., "Pulse wave analysis," $\mathrm{Br}$ J Clin Pharmacol, vol. 51, pp. 507-522, 2001.

[18] F. Scholkmann et al., "An efficient algorithm for automatic peak detection in noisy periodic and quasi-periodic signals," Algorithms, vol. 5, no. 4, pp. 599-603, Nov 2012.

[19] J. Y. Wagner et al., "Noninvasive continuous versus intermittent arterial pressure monitoring: evaluation of the vascular unloading technique (CNAP device) in the emergency department," Scand J Trauma Resusc Emerg Med, vol. 22, no. 8, Jan 2014.

Address for correspondence:

Tuukka Panula

Kiinamyllynkatu 10, 20520, Turku, Finland

tuukka.j.panula@utu.fi 